\title{
Prevalencia de fascioliasis en ovinos y bovinos en la provincia de Pataz, La Libertad, Perú, mediante examen coproparasitológico y Western Blot
}

\section{Prevalence of fascoliasis in sheep and cattle in the province of Pataz, La Libertad, Peru through coproparasitological tests and Western Blot}

\author{
César Jara Campos ${ }^{1,2}$, Hermes Escalante Añorga ${ }^{1}$, Wilson Cassana ${ }^{1}$, \\ Kelly Davelois Atac ${ }^{1}$, Adderly Benites Murrieta ${ }^{1}$
}

\section{Resumen}

\begin{abstract}
El estudio tuvo como objetivo determinar la prevalencia de fascioliasis en ovinos y bovinos en cinco distritos de la Provincia de Pataz, La Libertad, Perú, mediante examen coprológico y western blot. El estudio fue de tipo transversal prospectivo. Se evaluaron muestras de heces y de sangre de 388 ovinos y 365 bovinos de los distritos de Buldibuyo, Chillia, Huaylillas, Taurija y Urpay, entre abril y octubre de 2015 por los métodos de formol-éter y Kato Katz y de Western Blot. El 61.8\% de ovinos y el 86.8\% de bovinos presentaron anticuerpos anti-Fasciola hepatica (serología positiva) y el $42.3 \%$ de los ovinos y el $62.4 \%$ de los bovinos presentaron huevos de $F$. hepatica en heces (coprología positiva). Los distritos de Buldibuyo, Chillia y Huaylillas presentaron las mayores prevalencias de fascioliasis, tanto en ovinos como en bovinos. Se concluye que la provincia de Pataz puede ser considerada zona hiperendémica a fasciolosis por Fasciola hepatica.
\end{abstract}

Palabras clave: fascioliasis; prevalencia; ganado; Western blot

\section{Abstract}

The aim of this study was to determine the prevalence of fascioliasis in sheep and cattle in five districts of the Province of Pataz, La Libertad, Peru, through coprological tests and Western blot. The study was a prospective cross-sectional type. Faecal and blood samples from 388 sheep and 365 cattle were evaluated from the districts of Buldibuyo, Chillia, Huaylillas, Taurija and Urpay, between April and October 2015 by the

\footnotetext{
${ }^{1}$ Laboratorio de Helmintología, Facultad de Ciencias Biológicas, Universidad Nacional de Trujillo, La Libertad, Perú

${ }^{2}$ E-mail: Cesarj75@hotmail.com
}

Recibido: 29 de marzo de 2018

Aceptado para publicación: 15 de septiembre de 2018 
methods of formalin-ether and Kato Katz and Western Blot. Results showed than 61.8\% of sheep and $86.8 \%$ of cattle presented anti-Fasciola hepatica antibodies (positive serology) and $42.3 \%$ of sheep and $62.4 \%$ of bovines presented eggs of $F$. hepatica in faeces (positive coprology). The districts of Buldibuyo, Chillia and Huaylillas presented the highest prevalence of fascioliasis in both ovine and bovine. It is concluded that the province of Pataz can be considered hyperendemic zone to fasciolosis by Fasciola hepatica.

Key words: fascioliasis; prevalence; cattle; Western blotting

\section{INTRODUCCIÓN}

La fascioliasis es una zoonosis parasitaria causada por Fasciola hepatica, tremátodo que afecta a animales mamíferos herbívoros y, eventualmente, al hombre (Escalante et al., 2011). F. hepatica tiene una distribución mundial, predominando en zonas templadas (Morales y Espino, 2012; Martins et al., 2014). Esta enfermedad es transmitida por especies de caracoles del género Lymnaea a través del consumo de verduras crudas contaminadas con las fases infectantes, las metacercarias (Villegas et al., 2012).

En la actualidad, el Perú está seriamente afectado por este trematodo, considerándose como zona hiperendémica, tanto para los seres humanos como los animales, con una alta prevalencia de infección en niños, que puede llegar hasta el 70\% (López et al., 2012). La fascioliasis animal está ampliamente distribuida en 21 de los 24 departamentos del Perú, dato conocido por los informes de decomisos de vísceras parasitadas en los mataderos bajo inspección de Servicio Nacional de Sanidad Agraria (SENASA, 2007), reportándose pérdidas anuales no menor a los US\$ 50 millones por el descarte de animales y decomiso de órganos infectados (Espinoza et al., 2010; Escalante et al., 2011).

En el Perú, la transmisión ocurre principalmente en las poblaciones rurales dedicadas a la agricultura en los valles y pendientes andinas hasta los $4500 \mathrm{msnm}$ (Leguía, 1997; Mas-Coma et al., 1999a), e incluso en zonas urbanas que rodean la ciudad capital de Lima y zonas cerca de la costa del Pacífico (Bargues et al., 2012).

Los focos de transmisión más importantes de fascioliasis son el valle de Cajamarca (2670 msnm), las áreas de Huertas-Julcán (3420 msnm) en el valle del Mantaro y la Irrigación Asillo (3909 msnm) en la Región Puno, con prevalencias de 24,21 y $26 \%$, respectivamente (Leguía, 1997; Náquira, 2000; Espinoza et al., 2010). La región La Libertad es una de las zonas con mayor población de ganado ovino y altamente endémica para fascioliasis, ocupando el tercer puesto en el Perú en cuanto al impacto negativo, dado que de los 30 mil animales beneficiados se llegaron a decomisar más de 14 mil hígados, lo que significa una tasa regional del $45.9 \%$ (Espinoza et al., 2010).

Reportes de seroprevalencia de fascioliasis en la Libertad son escasos. En Santiago de Chuco se reportó prevalencia entre 7 y $22 \%$ a nivel distrital (Ayala et al., 2008), $12.9 \%$ en Mollepata, $10.8 \%$ en Mollebamba, $7.8 \%$ en Agallpampa, $14.5 \%$ en Huamachuco y $7.4 \%$ en Usquil (Ruiz et al., 2014) no habiéndose encontrado reportes para los distritos de la provincia de Pataz.

El diagnóstico de la fascioliasis se hace convencionalmente por el hallazgo de huevos en heces; sin embargo, esta técnica tiene poca sensibilidad debido a la poca cantidad 
de huevos que elimina el parásito. Se ha comprobado la eficiencia de la técnica de Western blot como apoyo al diagnóstico clínico (Cruz et al., 2007; Saavedra et al., 2010). Por ello, se ha propuesto al inmunodiagnóstico como alternativa, que permite la detección de anticuerpos, tanto en la fase aguda como crónica de la enfermedad. En el Perú se utilizan dos técnicas serológicas: Fas-2 ELISA y la electroinmunotransferencia o Western blot, con una sensibilidad de $92-100 \%$ y una especificidad de 84-100\%, (Escalante et al., 2011; Shafiei et al., 2015). Por estas razones, el objetivo del presente estudio fue determinar la prevalencia de fascioliasis en ganado ovino y bovino en cinco distritos de la provincia de Pataz, La libertad.

\section{MATERIALES Y MéTOdOS}

\section{Lugar de Estudio}

Se realizó un estudio descriptivo de corte transversal (encuesta de prevalencia) entre abril y octubre de 2015 en la zona central de la provincia de Pataz; específicamente en los distritos de Buldibuyo (Anexos Nuevo Porvenir y Llampao, $3162 \mathrm{msnm}$ ), Chillia (Anexos Nunamarca y Shacana, $3118 \mathrm{msnm}$ ), Huaylillas (2500 msnm), Taurija (Anexo: Huayao, $3111 \mathrm{msnm}$ ), y Urpay (Anexos Macania y Olgoyaco, $2688 \mathrm{msnm}$ ). La Provincia de Pataz está ubicada en la parte oriental de la Región La Libertad, Perú (Figura 1), presenta un clima variado, con humedad relativa de $71-97 \%$ y temperatura ambiente media entre 2 y $20{ }^{\circ} \mathrm{C}$ y con mayor frecuencia de lluvias entre octubre y abril (INEI, 2012; Región La Libertad, 2015). Este régimen de lluvias permite el crecimiento de plantas de tallo corto que sirven de alimento a ovinos, bovinos y caprinos. Los animales usualmente pastan durante el día y son encerrados por las noches en rediles que circundan los domicilios.

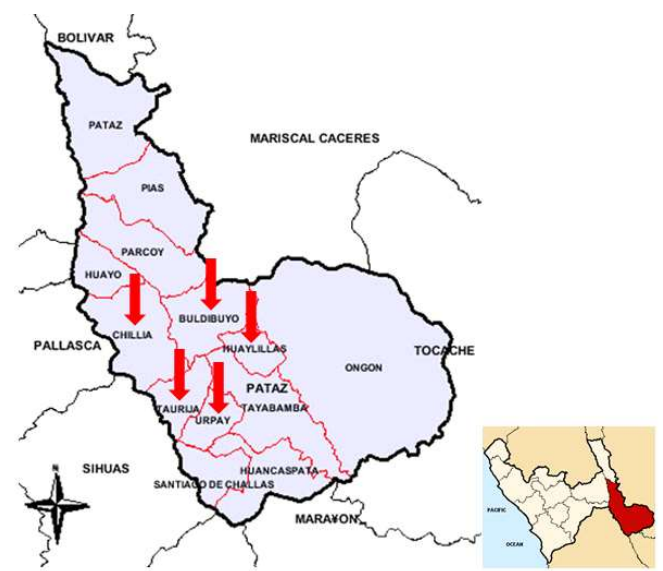

Figura 1. Mapa político de la provincia de Pataz, Región La Libertad, Perú, mostrando los cinco distritos bajo estudio (flechas color rojo). Fuente: http://www.peruto-ptours.com/ index12pa_mapa_Provincia_-pataz.html

La zona de muestreo se determinó utilizando un muestreo no probabilístico basado en las características ecológicas tipificadas como pequeños valles $u$ hondonadas donde se lleva a cabo la crianza de ganado al pastoreo.

\section{Población y Muestras}

Se tuvo una población de 388 ovinos, de los cuales se tomó una muestra de sangre y heces a 346 ovinos y a 42 solo se logró obtener la muestra de heces; mientras que se contó con 365 bovinos de los cuales a 306 se les tomó muestra de sangre y heces y a 59 solo la muestra de sangre. Se trabajó con animales cuyos propietarios aceptaron participar en el estudio (Cuadro 1). Los animales muestreados no habían recibido tratamiento antiparasitario alguno.

Para el análisis coprológico, las muestras se recolectaron directamente del recto de los animales (aproximadamente $10 \mathrm{~g}$ ) en frascos de polietileno, conservadas en formol al $10 \%$ para su transporte y trasladadas en el 
Cuadro 1. Muestras analizadas de ovinos y bovinos para determinar la presencia de anticuerpos anti-Fasciola hepatica en suero (análisis serológico) y de huevos de este helminto (análisis coprológico) en heces en distritos de la provincia de Pataz, La Libertad, Perú

\begin{tabular}{lcccc}
\hline & \multicolumn{2}{c}{ Serológico } & \multicolumn{2}{c}{ Coprológico } \\
\cline { 2 - 5 } & $\begin{array}{c}\text { Ovinos } \\
(\mathrm{n})\end{array}$ & $\begin{array}{c}\text { Bovinos } \\
(\mathrm{n})\end{array}$ & $\begin{array}{c}\text { Ovinos } \\
(\mathrm{n})\end{array}$ & $\begin{array}{c}\text { Bovinos } \\
(\mathrm{n})\end{array}$ \\
\hline Buldibuyo (N. Porvenir y Llampao) & 97 & 98 & 102 & 76 \\
Chillia (Nunamarca y Shacana) & 13 & 39 & 87 & 51 \\
Huaylillas & 104 & 103 & 79 & 62 \\
Taurija (Huayao) & 32 & 39 & 29 & 36 \\
Urpay (Macania y Olgoyaco) & 100 & 86 & 91 & 81 \\
\hline Total & 346 & 365 & 388 & 306 \\
\hline
\end{tabular}

término de cinco días al Laboratorio de Helmintología Parasitaria del Departamento de Microbiología y Parasitología, Facultad de Ciencias Biologías, Universidad Nacional de Trujillo (Trujillo). Para el análisis sanguíneo, las muestras de sangre se extrajeron de la vena femoral interna en el caso de los ovinos y de la vena yugular en el caso de los bovinos. En ambos casos se utilizaron tubos tipo vacutainer conteniendo gel separador y activador de la coagulación. La sangre se centrifugó a $986 \mathrm{~g}$ durante 15 min y almacenados a $-20^{\circ} \mathrm{C}$.

\section{Análisis de Laboratorio}

Las muestras fecales fueron examinadas mediante la técnica de Kato-Katz cualitativa (Beltrán et al., 2003), y la técnica de Ritchie (técnica formol-éter) para la detección de huevos de $F$. hepatica (Beltrán et al., 2003; Delgado, 2012).

Los sueros fueron analizados mediante la técnica serológica de Western blot para la detección de anticuerpos tipo IgG contra Fasciola hepatica, utilizando los protocolos desarrollados en el laboratorio (Escalante et al., 2011).

\section{Análisis Estadístico}

Se estimó la frecuencia relativa porcentual de la enfermedad con base al número de muestras positivas obtenidas y se expresó en porcentaje. Se determinó la asociación de las prevalencias serológicas y coprológicas de la infección por $F$. hepatica, tanto en ovinos como en bovinos; así como de las prevalencias encontradas por distrito mediante la prueba estadística Test de Proporciones, con un valor de significancia de $\mathrm{p}<0.05$. Aspectos Éticos

Los sueros utilizados en esta investigación fueron obtenidos previo asentimiento informado de los productores y procesados en condiciones de anonimato. La Comisión de Ética de la Facultad de Ciencias Biológicas, segun Acta de Reunión N. ${ }^{\circ} 002-2015$ acuerda que el Proyecto Canon $N^{\circ}$ 004-2013 reune las consideraciones éticas establecidas en el Reglamento del Comité de Ética en Investigación de la Universidad Nacional de Trujillo por RCU N. ${ }^{\circ} 268-2012 / \mathrm{UNT}$. 
Cuadro 2. Prevalencia de la infección por Fasciola hepatica en ovinos determinado mediante la técnica serológica Western blot y las técnicas coprológicas Kato-Katz cualitativo y de Ritchie, en cinco distritos de la Provincia de Pataz, La Libertad, Perú (abril a octubre, 2015)

\begin{tabular}{lcccc}
\hline \multirow{2}{*}{ Distritos } & \multicolumn{2}{c}{ Suero (examen serológico) } & \multicolumn{2}{c}{ Heces (examen coprológico) } \\
\cline { 2 - 5 } & Muestras (n) & Positivos (\%) & Muestras (n) & Positivos (\%) \\
\hline Buldibuyo & 97 & $76.3^{\mathrm{a}}$ & 102 & $30.4^{\mathrm{a}}$ \\
Chillia & 13 & $15.4^{\mathrm{b}}$ & 87 & $29.9^{\mathrm{b}}$ \\
Huaylillas & 104 & $68.3^{\mathrm{a}}$ & 79 & $62.0^{\mathrm{c}}$ \\
Taurija & 32 & $6.2^{\mathrm{b}}$ & 29 & $3.4^{\mathrm{d}}$ \\
Urpay & 100 & $65.0^{\mathrm{a}}$ & 91 & $62.6^{\mathrm{c}}$ \\
\hline Total & 346 & 61.8 & 388 & 42.3 \\
\hline
\end{tabular}

Valores con diferente superíndice dentro de columnas difieren significativamente $(p<0.05)$

Cuadro 3. Prevalencia de la infección por Fasciola hepatica en bovinos Bos taurus determinado mediante la técnica serológica Western blot y las técnicas coprológicas Kato-Katz cualitativo y de Ritchie, en cinco distritos de la Provincia de Pataz, La Libertad, Perú (abril a octubre, 2015)

\begin{tabular}{lcccc}
\hline \multirow{2}{*}{ Distritos } & \multicolumn{2}{c}{ Suero (examen serológico) } & \multicolumn{2}{c}{ Heces (examen coprológico) } \\
\cline { 2 - 5 } & Muestras (n) & Positivos (\%) & Muestras (n) & Positivos (\%) \\
\hline Buldibuyo & 98 & $100.0^{\mathrm{a}}$ & 76 & $77.6^{\mathrm{a}}$ \\
Chillia & 39 & $51.3^{\mathrm{b}}$ & 51 & $60.8^{\mathrm{a}}$ \\
Huaylillas & 103 & $100.0^{\mathrm{a}}$ & 62 & $69.3^{\mathrm{a}}$ \\
Taurija & 39 & $56.4^{\mathrm{b}}$ & 36 & $41.7^{\mathrm{a}}$ \\
Urpay & 86 & $86.0^{\mathrm{c}}$ & 81 & $53.1^{\mathrm{a}}$ \\
\hline Total & 365 & 86.8 & 306 & 62.4 \\
\hline
\end{tabular}

Valores con diferente superíndice dentro de columnas difieren significativamente $(p<0.05)$

\section{Resultados}

Se encontró una seroprevalencia de fascioliasis en ganado ovino de $61.8 \%$ $(214 / 388)$ y de $86.8 \%$ en ganado bovino (317/365) mediante la técnica de Western blot. Así mismo, de 42.3\% (164/388) y de $62.4 \%$ (191/365) en ganado ovino y bovino, respectivamente, mediante la técnica coproparasito-lógica (Cuadros 2 y 3 ).
En ovinos, los distritos de Buldibuyo, Huaylillas y Urday presentaron las prevalencias más elevadas mediante el análisis serológico, mientras que Huaylillas y Urpay lo fueron mediante el análisis coprológico. En este mismo sentido, en el caso de bovinos, los distritos de Buldibuyo y Huaylillas presentaron la mayor prevalencia mediante el análisis serológico, mientras que no hubo diferencias entre distritos mediante el análisis coprológico (Cuadros 2 y 3 ). 


\section{Discusión}

El análisis serológico se basó en que antígenos de excreción-secreción (E/S) de las fases inmaduras y maduras de $F$. hepatica son los primeros en inducir la respuesta inmune humoral en casos de infecciones (Espino et al., 2000; Escalante et al., 2011). Los anticuerpos específicos se producen tempranamente contra los antígenos de $\mathrm{E} / \mathrm{S}$, permitiendo la detección en la fase aguda (dos semanas después de la infección)., pudiendo persistir por largos periodos (Timoteo et al., 2005). La técnica de electroinmunotransferencia (EITB) o Western blot para la detección de la fascioliasis presenta una sensibilidad de $95.5 \%$ y una especificidad de $100 \%$ (Escalante et al., 2011). Sin embargo, existen limitaciones de las pruebas serológicas, en tanto que no distinguen entre infección actual o pasada y, por tanto, un resultado positivo en un ovino o bovino proveniente de una zona endémica para fascioliasis, como lo es la provincia de Pataz, puede deberse a una infección reciente o el resultado de una respuesta inmune a la infección en el pasado.

La técnica de sedimentación formol-éter y la técnica de Kato-Katz, utilizados en este estudio para el análisis coprológico solo detectan los casos en la fase crónica de la infección, pues en ese estado, el parásito se encuentra ubicado en las vías biliares, ha alcanzado la madurez sexual y emite huevos que son excretados en las heces. Por lo tanto, estas técnicas no detectan la fase aguda o la fascioliasis ectópica (Espinoza et al., 2010).

Los resultados obtenidos en el presente estudio, tanto para ovinos como bovinos, presentaron diferencias significativas entre los resultados coprológicos y serológicos $(\mathrm{p}<0.05)$. No obstante, estos resultados ubican a la Provincia de Pataz como una zona hiperendémica para la fascioliasis, según la clasificación epidemiológica de la Organización Mundial de la Salud (OMS), propuesta por Mas-Coma (1999b).
Las mayores prevalencias en bovinos en comparación con ovinos podrían deberse a que los primeros viven mayor tiempo, teniendo más opción de tener contacto con las formas infectantes (Valderrama, 2016). Ajuzgar por lo investigado en otras regiones del Perú y en otros países, la fascioliasis en ovinos y vacunos se presenta con elevadas frecuencias, probablemente debido al tipo de alimento consumido, especialmente cuando la crianza se hace en pastizales abiertos, naturales o sembrados con alfalfa u otras gramíneas. En efecto, en Vilcashuamán (Ayacucho) se detectó $52.1 \%$ de prevalencia en ovinos y $47.6 \%$ en bovinos (Ticona et al., 2010) y de $23.1 \%$ en bovinos de Huancavelica (Valencia et al., 2005). Del mismo modo, en Brasil se detectó el 14.4\% en bovinos (Bennema et al., 2014) y el $72 \%$ en bovinos de Vietnam (Nguyen et al., 2011).

Los distritos de Buldibuyo, Huaylillas, Taurija y Urpay muestran prevalencias serológicas mayores que las prevalencias coprológicas, tanto en ovinos como en bovinos. La explicación radica en la ocurrencia de animales recién infectados, que hayan tenido una infección en el pasado, pero también a que la detección de anticuerpos contra F. hepatica no es específico. Al tratarse de antígenos muy complejos, pueden hacer epítopos compartidos, lo que contribuye a que se presenten reacciones cruzadas con otras patógenos.

En el distrito de Chillia se presentaron, no obstante, resultados contradictorios. Este fue el primer distrito muestreado, obteniéndose prevalencias coprológicas mayores que las serológicas en las dos especies. Esto pudo deberse a la falta de experiencia en la toma de muestras, llegándose a obtener una mayor cantidad de muestras de heces que muestras de suero. Lamentablemente no pudo hacerse un segundo muestreo pues los animales fueron tratados con antihelmínticos como parte de las campañas de dosificación de la municipalidad distrital. 
Los factores climáticos, como la precipitación y la temperatura, influencian la distribución y prevalencia de la fascioliasis (Novobilský et al., 2015), pues afectan la supervivencia y la transmisión de los huéspedes intermediarios (caracoles del género Lymnaea) y las fases larvarias de F. hepatica (Malone y Zukowski, 1992; Fox et al., 2011). Los distritos de Buldibuyo, Chillia y Taurija presentan climas semisecos, semifríos y húmedos, mientras que los de Huaylillas y Urpay tienen climas lluviosos, cálidos y húmedos, lo cual se ha visto reflejado en los porcentajes de prevalencias encontrados. No obstante la importancia de los factores climáticos, los factores ambientales también tienen gran importancia en la fascioliasis (Charlier et al., 2011; Bennema et al., 2011; Novobilský et al., 2015). Esto se confirma con los resultados obtenidos en el distrito de Buldibuyo, distrito que presenta terrenos de menor pendiente y con una mayor presencia de bofedales y afluentes de agua que favorecen el desarrollo de los hospederos intermediarios de F. hepatica.

Todas las muestras de los distritos de Buldibuyo y Huaylillas presentaron anticuerpos anti-Fasciola hepatica. Esto podría deberse a que son zonas más propicias para el desarrollo del caracol hospedero intermediario, presentan un río a lo largo de todo el distrito haciéndose más factible la formación de charcos, lugares donde desarrolla el caracol.

\section{Conclusiones}

- La prevalencia serológica de Fasciola hepatica en cinco distritos de la provincia de Pataz, La Libertad, fue de 61.8 y $42.3 \%$ en ovinos y de 86.8 y $62.4 \%$ en bovinos mediante serología (Western blot) y análisis coprológico (Kato-Katz cualitativo y de Ritchie), respectivamente.

- Los resultados demuestran que la zona central de la provincia de Pataz es una zona hiperendémica de infección por $F$. hepatica en ovinos y bovinos.

\section{Literatura Citada}

1. Ayala MS, Bustamante S, González M. 2008. Estado actual de la fascioliasis en Mollebamba, Santiago de Chuco, Región La Libertad y su abordaje por niveles de atención y prevención. Rev Med Vallejiana 5: 89-99.

2. Bargues MD, Artigas $P$, Khoubbane M, Ortiz P, Naquira C, Mas-Coma S. 2012. Molecular characterisation of Galba truncatula, Lymnaea neotropica and L. schirazensis from Cajamarca, Peru and their potential role in transmission of human and animal fascioliasis. Parasite Vector 5: 174. doi: 10.1186/1756-3305-5-174

3. Beltrán M, Tello CR, Náquira VC. 2003. Manual de procedimientos de laboratorio para el diagnóstico de los parásitos intestinales del hombre (Serie de Normas Técnicas $\left.N^{\circ} 37\right)$. Lima, Perú: Instituto Nacional de Salud. 90 p.

4. Bennema SC, Ducheyne $E$, Vercruysse $J$, Claerebout $E$, Hendrickx G, Charlier J. 2011. Relative importance of management, meteorological and environmental factors in the spatial distribution of Fasciola hepatica in dairy cattle in a temperate climate zone. Int J Parasitol 41: 225-233. doi: 10.1016/j.ijpara.2010.09.003

5. Bennema SC, Scholte RGC, Molento MB, Medeiros C, Carvalho O. 2014. Fasciola hepatica in bovines in Brazil: data availability and spatial distribution. Rev Inst Med Trop Sp 56: 35-41. doi: 10.1590/S0036-46652014000100005

6. Charlier J, Bennema SC, Caron Y, Counotte M, Ducheyne E, Hendrickx G, Vercruysse J. 2011. Towards assessing fine-scale indicators for the spatial transmission risk of Fasciola hepatica in cattle. Geospatial Health 5: 239-245. doi: 10.4081/gh.2011.176

7. Cruz O, Pérez MT, Lubián AL, Montano L, Silva E. 2007. Fluido oral y orina como muestras alternativas en el diagnóstico confirmatorio de la infección 
por VHI-1. Rev Cubana Med Trop 59: 134-138.

8. Delgado N, Sierra R, Espinoza C. 2012. Comparación de las técnicas Kato-Katz, TSET y TSR en el diagnóstico de infección por Fasciola hepatica en humanos. Rev Univ Ind Santander Salud 44: 7-12

9. Escalante H, Davelois K, Ortíz P, Rodríguez H, Díaz E, Jara C. 2011. Estandarización de la técnica de Western blot para el diagnóstico de la fascioliasis humana utilizando antígenos de excreción-secreción de Fasciola hepatica. Rev Peru Med Exp Salud Pública 28: 454-461.

10. Espino AM, Borges A, Duménigo BE. 2000. Coproantígenos de Fasciola hepatica de posible utilidad en el diagnóstico de la fascioliasis. Rev Panam Salud Publ 7: 225-231.

11. Espinoza JR, Terashima A, HerreraVelit P, Marcos LA. 2010. Fascioliasis humana y animal en el Perú: impacto en la economía de las zonas endémicas. Rev Peru Med Exp Salud Pública 27: 604612.

12. Fox NJ, White PCL, McClean CJ, Marion G, Evans A, Hutchings MR. 2011. Predicting impacts of climate change on Fasciola hepatica risk. PLos One 6: e16126. doi: 10.1371/journal.pone. 0016126

13. [INEI] Instituto Nacional de Estadística e Informática. 2012. La Libertad. Compendio estadístico 2012. [Internet]. Disponible en: https://www.inei.gob.pe/ $\mathrm{media/M}$ e nu Recursivo/ publicaciones_digitales/Est/Lib1060/ libro.pdf

14. Leguía G. 1997. Acute and subacute fascioliasis of alpacas (Lama pacos) and treatment with triclabendazol. Trop Anim Health Pro 29: 31-32.

15. López M, White AC, Cabada MM. 2012. Burden of Fasciola hepatica infection among children from Paucartambo in Cusco, Peru. Am J Trop Med Hyg 86: 481-485. doi: 10.4269/ ajtmh.2012.11-0448
16. Malone JB, Zukowski SH. 1992. Geographic models and control of cattle liver flukes in the Southern USA. Parasitol Today 8: 266-270. doi: 10.1016/ 0169-4758(92)90138-R

17. Marcos L, Maco V, Terashima A, Samalvides $F$, Miranda E, Tantaleán $M$, et al. 2004. Hiperendemicidad de fasciolosis humana en el Valle del Mantaro, Perú: factores de riesgo de la infección por Fasciola hepatica. Rev Gastroenterol Peru 24: 158-164.

18. Martins IV, Avelar BR, Bernardo CD, Leão AC, Salim MJ. 2014. Distribution of bovine fascioliasis and associated factors in south Espírito Santo, Brazil: an update. Rev Bras Parasitol Vet 23: 2329. doi: 10.1590/S1984-29612014003

19. Mas-Coma S, Anglés R, Esteban JG, Bargues MD, Buchon P, Franken M, Strauss W. 1999a. The Northern Bolivian Altiplano: a region highly endemic for human fascioliasis. Trop Med Int Health 4: 454-467. doi: 10.1046/ j.1365-3156.1999.00418.x

20. Mas-Coma MS, Esteban JG, Bargues MD. 1999b. Epidemiology of human fascioliasis: a review and proposed new classification. Bull World Health Organ 77: 340-346.

21. Morales A, Espino AM. 2012. Evaluation and characterization of Fasciola hepatica tegument protein extract for serodiagnosis of human fascioliasis. Clin Vaccine Immunol 19: 1870-1878. doi: 10.1128/CVI.00487-12

22. Náquira VC. 2000. Epidemiology of fascioliasis in Latin America. En: XVth International Congress for Tropical Medicine and Malaria. Cartagena, Colombia.

23. Nguyen TG, Le TH, Dao TH, Tran TL, Praet N, Speybroeck N, Vercruysse J, Dorny P. 2011. Bovine fascioliasis in the human hyperendemic Binh Dinh province in Central Vietnam. Acta Trop 117: 1922. doi: 10.1016/j.actatropica.2010.09.003

24. Novobilský A, Novák J, Björkman C, Höglund J. 2015. Impact of meteorological and environmental factors 
on the spatial distribution of Fasciola hepatica in beef cattle herds in Sweden. BMC Vet Res 11:128. doi: 10.1186/ s12917-015-0447-0

25. Region La Libertad. 2015. Pataz. [Internet]. Disponible en: http:// maravillosaregionlalibertad.blogspot.com/ 2015/06/pataz.html

26. Ruiz W, Asmat PE, Peña HH, Ramírez R, Ramírez R, Lezama $P$. 2014. Diagnóstico serológico y coproparasitoscópico de Fasciola hepatica en los adolescentes escolares de los distritos de Agallpampa, Usquil, Huamachuco, Mollebamba y Mollepata. La Libertad, enero-noviembre 2014. Pueblo Cont 25: 15-21.

27. Saavedra H, Gonzales, Alvarado M, Porras M, Vargas V, Cjuno RA, Garcia $H H$, et al. 2010. Diagnóstico y manejo de la neurocisticercosis en el Perú. Rev Peru Med Exp Salud Publica 27: 586591.

28. [SENASA] Servicio Nacional de Sanidad Agraria. 2007. Estrategias de intervención para la prevención y control de fasciolasis causada por Fasciola hepatica. Cajamarca: SENASA.

29. Shafiei R, Sarkari B, Sadjjadi SM.2015. Performance of a $27 \mathrm{kDa}$ Fasciola hepatica antigen in the diagnosis of human fascioliasis. J Lab Physicians 7: 17-20. doi: 10.4103/09742727.154781
30. Ticona D, Chávez A, Casas G, Chavera, Li O. 2010. Prevalencia de Fasciola hepatica en bovinos y ovinos de Vilcashuamán, Ayacucho. Rev Inv Vet Peru 21: 168-174.

31. Timoteo O, Maco V, Neyra V, Yi PJ, Leguía G, Espinoza JR. 2005. Characterization of the humoral immune response in alpacas (Lama pacos) experimentally infected with Fasciola hepatica against cysteine proteinases Fas 1 and Fas2 and histopathological findings. Vet Immunol Immunop 106: 77 86. doi: 10.1016/j.vetimm.2005.01.007

32. Valderrama AA. 2016. Prevalencia de fascioliasis en animales poligástricos de Perú, 1985-2015. Rev Med Vet 32: 121129. doi: $10.19052 / \mathrm{mv} .3861$

33. Valencia N, Pariona A, Huamán M, Miranda F, Quintanilla S, González A. 2005. Seroprevalencia de fascioliasis en escolares y en ganado vacuno de la provincia de Huancaveliva, Perú. Rev Perú Med Exp Salud Pública 22: 96-102.

34. Villegas F, Angles R, Barrientos R, Barrios $G$, Valero $M A$, Hamed $K$, Grueninger $\mathrm{H}$, et al. 2012. Administration of triclabendazole is safe and effective in controlling fascioliasis in an endemic community of the Bolivian Altiplano. Plos Neglect Trop D 6: e1720. doi: 10.1371/journal.pntd.0001720 